\title{
The Influence of Day 2 Blastomere Symmetry on Blastocyst Grade and Ploidy Status
}

\author{
Diana Chieh Xing Tain ${ }^{1, \star}$, Michelle Sheng Rong Lim ${ }^{1}$, Bee Lian $\mathrm{Ng}^{1}$, Elizabeth Hammond ${ }^{2}$, Pak Seng Wong ${ }^{1}$ \\ ${ }^{1}$ Sunfert International Fertility Centre, Unit 2-2, Level 2, Nexus, Bangsar South, 7, Jalan Kerinchi, 59200 Kuala Lumpur, Malaysia \\ ${ }^{2}$ Fertility Associates, Level 3, Ascot Central, 7 Ellerslie Racecourse Drive, Private Bag 28910, Remuera, Auckland 1051, New Zealand
}

\begin{abstract}
Previous studies have suggested that aneuploidy rates are co-related with cell asymmetry at the cleavage stage. A retrospective study was carried out to determine the significance of blastomere symmetry at the 4-cell stage on blastocyst grade and ploidy status.

732 Day 5/6 blastocysts from 191 patients undergoing Pre-implantation Genetic Testing for Aneuploidy were analysed with time-lapse imaging (Embryoscope, Vitrolife) during 2017. Blastomere symmetry was measured at the first image of 4-cells on Day 2 by tabulating the mean diameter of 2 lines drawn perpendicularly on each blastomere.

Symmetry was defined as the blastomere diameter difference of $\leq 25 \%$. Trophectoderm (TE) biopsy was performed on Day $5 / 6$ followed by chromosomal evaluation using Next Generation Sequencing (VeriSeq Protocol, Illumina). Blastocyst grade was classified as either "Good" (inner cell mass (ICM) and TE, AA respectively), "Fair/Good" (AB, BA), "Fair" (BB) and "Poor" (early blastocyst grade 2 or TE grading of $\mathrm{C}$ ). The significance of blastomere symmetry on blastocyst grade and ploidy status was measured using chi-square tests.

There was no significance difference in resulting blastocyst quality for symmetrical and asymmetrical embryos (Table 1: $\mathrm{p}=$ 0.10). Furthermore, there was no significance difference in the euploid rate $(42.5 \%$ vs. $45.3 \%)$ or mosaic rate $(22.1 \%$ vs. $16.2 \%)$ between symmetrical and asymmetrical embryos $(\mathrm{p}=0.24)$.

In conclusion, the presence of asymmetrical blastomeres at the 4-cell stage do not impact the good quality blastocyst formation rate and euploidy rate for embryos that progress into blastocysts. However, this study excludes embryos that do not develop to the blastocyst stage and those with erratic division patterns, direct cleavage and reverse cleavage on Day 2, both of which have potential to influence ploidy result.

Asymmetrical 4-cell embryos have the potential for high quality euploid blastocyst progression and can be considered for day 2 embryo transfer in the absence of symmetrical 4-cell embryos.
\end{abstract}

Keywords: Day 2; 4-Cell Embryo; Symmetry; Asymmetry; Even; Uneven.

\section{INTRODUCTION}

Blastomere symmetry at the cleavage stage (even or uneven) has been an important grading parameter alongside other observations such as cell number, cellular fragmentation and multinucleation. For blastomere symmetry, an optimal Day 1 (26-28 hours) embryo is defined as having 2 blastomeres of equal size following the first mitotic division, Day 2 (44 hours) embryo having 4 blastomeres of equal size arranged in a tetrahedron shape, and an optimal Day 3 (68 hours) embryo having 8 blastomeres of equal size (Alpha Scientists in Reproductive Medicine and ESHRE Special Interest Group of Embryology, 2011). The presence of blastomeres of unequal size is frequently observed in in-vitro embryos at the 2 , 4 and 8-cell stages signifying an unequal portioning of cytoplasm between daughter cells (Puissant et al., 1987; Basile et al., 2015). With an unequal portioning of cytoplasm between daughter cells, it is thought that there may be an unequal segregation of proteins,
mRNA and mitochondria which may impair development with the possible implication of chromosomal aberrations (Hardarson et al., 2001; Alpha Scientists in Reproductive Medicine and ESHRE Special Interest Group of Embryology, 2011).

It has been reported that the presence of uneven blastomeres in an embryo lower developmental potential. Hence, embryos with uneven blastomeres are less likely to be selected (de-selection) for transfer during conventional morphological assessment and time-lapse monitoring (Hardarson et al., 2001; Alpha Scientists in Reproductive Medicine and ESHRE Special Interest Group of Embryology, 2011; Basile et al., 2015). Although Day 2 embryos with uneven blastomeres have a $12 \%$ lower implantation rate compared to Day 2 embryos with even blastomeres, they still maintain a modest implantation rate of $24 \%$, demonstrating that normal development can ensue (Hardarson et al., 2001). For time-lapse monitoring, measuring blastomere symmetry has most recently become easier

() 2019 by the Asia Pacific Initiative on Reproduction (ASPIRE) and World Scientific Publishing Co. Pte. Ltd. $\odot$ Open Access article under the CC BY-NC-ND license (http://creativecommons.org/licenses/by-nc-nd/4.0/).

Received 20 February 2019; Accepted 4 June 2019; Published 28 June 2019

${ }^{*}$ Correspondence should be addressed to: Diana Tain Chieh Xing, Sunfert International Fertility Centre Unit 2-2, Level 2, Nexus, Bangsar South, 7 , Jalan Kerinchi, 59200 Kuala Lumpur, Malaysia. Email: diana.tain@sunfert.com. 
to quantify using measuring tools present within time-lapse monitoring software, where symmetry at the 2-cell stage has been incorporated into a known implantation data (KIDscore) algorithm during selection for Day 3 transfer (Basile et al., 2015).

Embryos with asymmetric blastomeres have been shown to have higher rates of aneuploidy at the cleavage stage compared to embryos with evenly sized blastomeres (Hardarson et al., 2001; Ziebe et al., 2003; Buehler et al., 2008; Shenoy et al., 2015). It has been shown, however, that embryos with asymmetric blastomeres that progress to the blastocyst stage may have a comparatively reduced rate of aneuploidy, which could be due to a higher rate of cleavage stage arrest for aneuploid embryos or the speculation that embryos may be able to exclude aneuploid cells arising from mitotic division to 'selfcorrect' during blastocyst progression (Barbash-Hazan et al., 2009; Bolton et al., 2016). Conversely, not all studies provide evidence for this (Shenoy et al., 2015).

Thus, it is useful to know the exact rate of aneuploidy for embryos that have uneven blastomeres but progress into useable blastocysts to confirm if blastomere asymmetry should be used for de-selecting embryos despite progression to the blastocyst stage. In this study, we focus on the relationship between blastomere asymmetry at the 4-cell stage, and the rate of aneuploidy in useable blastocysts, compared to those blastocysts that arise from even 4-cell embryos. Time-lapse monitoring was used to accurately quantify the size of the blastomeres for an objective assessment and downstream ploidy status was determined using trophectoderm (TE) biopsy and comprehensive chromosome screening with next-generation sequencing (NGS).

\section{MATERIALS AND METHODS}

\section{Study design}

A retrospective analysis was performed to compare the euploid, aneuploid and mosaic rate of useable blastocysts rising from either asymmetrical or symmetrical 4-cell embryos. Time-lapse monitoring was used to accurately quantify blastomere size by using the tools provided by the time-lapse software, and embryos showing other cleavage stage aberrations such as reverse, direct or erratic cleavage were excluded to remove confounding variables from the analysis. Useable blastocysts arising from either asymmetrical or symmetrical 4-cell embryos were classified into 4 categories according to their morphological grade (Good, Fair/Good, Fair and Poor) and ploidy status was assessed by TE biopsy and NGS. Implantation data was not investigated as the focus of the study was to determine the blastocysts ploidy rate from asymmetrical or symmetrical 4-cell embryos across a large cohort of embryos $(n=732)$.

\section{Patient selection}

This study was conducted on 732 Day 2 embryos which developed into useable blastocysts from 191 patients opting for Preimplantation Genetic Testing for Aneuploidy (PGT-A) from January 2017 to December 2017. Follicular growth from ovarian stimulation was monitored using trans-vaginal ultrasound and once the number of large follicles ( $>17 \mathrm{~mm}$ diameter) exceeded 3, human chorionic gonadotropin was administered for the final maturation of follicles. Oocyte recovery was guided under trans-vaginal ultrasound scanning conducted $36-37$ hours post-trigger.

\section{Embryo culture and grading}

All mature oocytes were subjected to intra-cytoplasmic sperm injection (ICSI) after 39-40 hours post-trigger and were subsequently cultured in continuous culture medium GT-L (Vitrolife, Sweden) for up to 6 days in the Embryoscope ${ }^{\text {tu }}$ (Vitrolife, Sweden). The oocytes were evaluated for fertilisation by the presence of two pronuclei at 16-18 hours post-injection (HPI) via Embryoviewer $^{\mathrm{mw}}$. All embryos were cultured to blastocyst stage for biopsy. Blastocysts were observed on Day 5 (112-114 HPI) and Day 6 (140 HPI) and were graded using the Gardner blastocyst morphology grading system (Gardner and Schoolcraft, 1999). Blastocyst morphology was classified into 4 categories according to the inner cell mass (ICM) and TE grade: Good (3-5 AA), Fair/ Good (3-5 AB, BA), Fair (3-6 BB), and Poor (early blastocyst grade 2 or grade $\mathrm{C}$ ). All normally fertilised useable blastocysts were included in this study with the exception of blastocysts arising from cleavage stage embryos with direct, reverse cleavage or other erratic cleavage patterns on Day 1 and/or 2 as they are confounding variables when investigating the relationship between blastomere symmetry and aneuploidy.

\section{Trophectoderm biopsy and preimplantation genetic testing for aneuploidies}

TE biopsy was performed on Day 5 or Day 6. An average of 5 TE cells were biopsied and the sample was amplified using Sureplex DNA Amplification System according to the manufacturer's protocol and analysed using NGS (Veriseq protocol, Illumina). Blastocysts were classified into 3 groups; Euploid, Mosaic and Aneuploid. Euploid blastocysts had no abnormalities detected or low levels of mosaicism $(<40 \%)$. Mosaic blastocysts had intermediate levels of mosaicism (40-70\%) while blastocysts with abnormalities detected and/or high mosaicism levels ( $\geq 75 \%$ ) are reported to be aneuploid. Following the biopsy, the blastocysts were vitrified in individual straws for future use.

\section{Blastomere symmetry}

Blastocysts that were biopsied were retrospectively analysed for blastomere symmetry and classified into two groups: symmetrical blastomeres and asymmetrical blastomeres (Fig. 1). Using embryoscope images, symmetry was measured at the first sign of a clear 4-cell division on Day 2 (44-46 HPI). Blastomere symmetry was defined as previously reported by Prados (2012); embryos with blastomeres with a diameter difference of $<25 \%$ were symmetrical while embryos with $\geq 25 \%$ diameter difference were asymmetrical. This was calculated by dividing the diameter of the smallest blastomere with that of the largest blastomere (Fig. 2). A value of $>0.75$ was considered symmetrical while a value of $\leq 0.75$ was asymmetrical. The $\geq 25 \%$ diameter difference being defined as asymmetrical represents unequal portioning of cytoplasm following embryo cell division which may impact downstream development but is clearly distinguished from cleavage stage fragmentation. The

Fig. 1. A symmetrically arranged 4-cell embryo (left). An asymmetrical 4-cell embryo with a large blastomere, B and a significantly smaller blastomere, A (right).

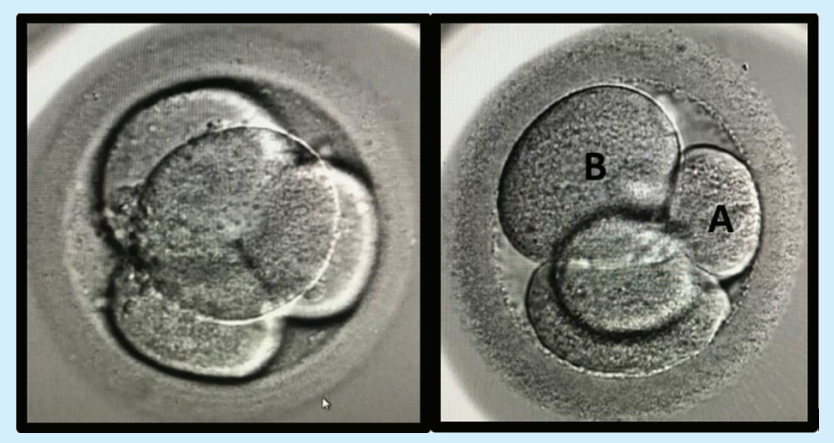


Fig. 2. Blastomere diameter measuring at $74 \mu \mathrm{m}$ (left) and $63 \mu \mathrm{m}$ (right). The blastomere has an average diameter of $68.5 \mu \mathrm{m}$.

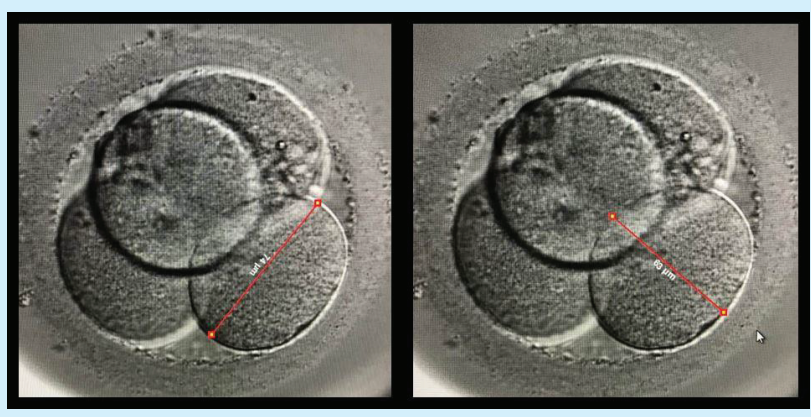

diameter of each of the 4 blastomeres was measured by tabulating the mean of 2 lines drawn perpendicularly (Fig. 2) using the tools provided on the Embryoviewer ${ }^{\text {Tix }}$ (Vitrolife, Sweden).

\section{Statistical analysis}

Chi-square tests were used to compare blastocyst quality and PGT-A results for blastocysts which developed from symmetrical and asymmetrical blastomeres on Day 2 (4-cell stage). T-tests were used to compare differences in patients' ages between study groups. Differences were considered statistically significant if $\mathrm{p}<0.05$.

\section{RESULTS}

A total of 732 blastocyst were included in the study with a majority of blastocysts arising from symmetrical blastomeres on Day $2(75.5 \%, n=553)$. The mean female age of the two groups of embryos did not differ significantly with the mean age of symmetrical blastomeres being 30.9 years and 31.4 years for asymmetrical blastomeres $(\mathrm{p}=0.4072)$. The patient age range in the whole cohort was $19-46$ years.

Among the blastocysts that were formed from symmetrical blastomeres, $14.3 \%(n=79)$ were good quality blastocysts, $40 \%$ were fair/good $(n=221), 44.7 \%$ were fair $(n=247)$ and $1.0 \%$ were poor $(n=6)$. For asymmetrical embryos, $16.8 \%(n=30)$ were good quality blastocysts, 30.2\% ( $\mathrm{n}=54)$ were fair/good, $52.5 \%(\mathrm{n}=94)$ were fair and $0.5 \%(n=1)$ were poor. The 'good' blastocyst formation rate did not differ significantly based on the symmetry of the blastomeres at the 4 -cell stage (14.3\% for symmetrical blastomeres and $16.8 \%$ for asymmetrical blastomeres) $(\mathrm{p}=0.1027)$.

The euploid rate and mosaic rate for blastocysts with symmetrical blastomeres were $42.5 \%(n=235)$ and $22.1 \%(n=122)$ respectively while for blastocyst with asymmetrical blastomeres, the rates were $45.3 \%(n=81)$ and $16.2 \%(n=29)$ respectively. The euploid rate and the mosaic rate did not differ significantly between blastocysts arising from symmetrical and asymmetrical 4-cell embryos $(\mathrm{p}=0.2405)$.

\section{DISCUSSION}

Our study primarily demonstrated a similar euploid rate between blastocysts arising from symmetrical (42.5\%) and asymmetrical (45.3\%) blastomeres. This suggests that if an asymmetrical embryo progresses to the blastocyst stage, then the chance of the embryo being euploid is no different compared to other embryos that had symmetrical blastomeres. Therefore, these results support the use of extended culture for asymmetric Day 2 embryos for Day 5 blastocyst transfers. If these embryos progress to the blastocyst stage, their rate of ploidy does not appear to be correlated to their asymmetrical cell division at the early cleavage stage.

Our findings conflict with earlier research which showed that embryos with unevenly sized blastomeres have a lower euploid rate compared to those with evenly sized blastomeres (Hardarson et al., 2001; Ziebe et al., 2003; Buehler et al., 2008; Shenoy et al., 2015). The aneuploid rate in uneven embryos ranged from $54.5 \%$ to $76.8 \%$ across various studies. One study employing a similar design to the current study demonstrated a $25 \%$ increase in aneuploidy rate following TE biopsy for embryos showing the highest degree of cell symmetry at the 4-cell stage versus those showing the lowest degree of cell symmetry, further finding that cell symmetry was more predictive that time-lapse parameters (Shenoy et al., 2015). The current study showed conflicting results for symmetry at the 4-cell stage, which may reflect differences in patient demographics, for example the patients in this study has a mean age of 31 , where high euploid rates are to be expected. Further differences in the rates between previous studies and the current study may possibly be attributed to the number of chromosomes tested and whether ploidy was tested at the cleavage stage (Hardarson et al., 2001; Ziebe et al., 2003; Buehler et al., 2008) or blastocyst stage (Shenoy et al., 2015). As aneuploidy is not limited to certain chromosomes, when the number of chromosomes tested increases, the aneuploidy rate concurrently increases. In Hardarson's, Ziebe's and Buehler's research, blastomere biopsy with FISH was carried out. This result is concurrent with other findings that the aneuploid and mosaic rate in the cleavage stage is higher than in the blastocyst stage (Northrop et al., 2010; Santos et al., 2010) as some aneuploid or mosaic embryos arrest at the cleavage stage. Lower implantation rates were shown when uneven embryos were utilized compared to embryos with even blastomeres with the speculation of abnormal ploidy status being a main contributor (Hardarson et al., 2001; Soydan et al., 2008). However, it is likely that poorer blastocyst progression from embryos with asymmetrical blastomeres was a factor.

Previous studies have used an inverted microscope to take multilayered photos of the blastomeres in the embryo (Vergouw et al., 2013), however a defining feature of the current study was the use of time-lapse to classify the embryos into the two groups based on their symmetry on Day 2. The definition of asymmetry used in the current study was a $\geq 25 \%$ diameter difference, which has been previously described (Prados, 2012). It is possible that a larger degree of asymmetry, for example a $\geq 50 \%$ diameter difference may have influence rate of aneuploidy, however few embryos progressing to the blastocysts stage fall into this category, therefore limiting an analysis based on a higher degree of asymmetry. The use of timelapse allows for objective measurements of the blastomere diameter at the first sign of cellular division producing 4 blastomeres at Day 2, we are able to accurately and objectively classify the embryos under undisturbed environment.

Blastocyst quality was also compared between blastocysts that originated from symmetrical and asymmetrical blastomeres. There was no significant difference found in the two groups, suggesting that symmetry at the 4-cell stage is not a predictor of blastocyst quality, provided that the embryo progresses to the blastocyst stage. A limitation of the current study is that only asymmetrical embryos that progressed to the blastocyst stage were included. As such, the blastulation rate and blastocyst utilization rate of symmetrical and asymmetrical embryos cannot be compared. The rate at which asymmetrical 4-cell embryos result in blastocyst formation should be confirmed in a larger study.

A possible explanation as to why uneven embryos are considered to have a role in poorer blastulation is the unequal cytoplasmic 
allocation to daughter cells. It can be theorised that uneven daughter cells cause unequal distribution of maternal transcripts (Wells et al., 2005) or proteins (Antczak and Van Blerkom, 1999) which could subsequently affect embryo development. Hence, it is logical to deduce that with asymmetrical blastomeres in the embryo, the chance of blastocyst formation would decline. Some studies have found that symmetry was not strongly correlated to the development potential of the embryo (Sjobolm et al., 2006). Thus, symmetry needs to be further evaluated on its effect on blastulation - however our study provides evidence, that in a relatively young patient cohort (mean age 31 years) the euploid rate is the same between blastocysts arising from symmetrical and asymmetrical 4-cell embryos, provided that they develop into useable blastocysts.

\section{CONCLUSION}

The presence of asymmetrical blastomeres at the 4-cell stage does not impact blastocyst quality and euploid rate for embryos that progress into useable blastocysts. Asymmetrical 4-cell embryos have the potential to produce high quality euploid blastocyst progress and can be considered for Day 2 embryo transfer in the absence of symmetrical 4-cell embryos.

\section{REFERENCES}

Alpha Scientists in Reproductive Medicine and ESHRE Special Interest Group of Embryology. The Istanbul consensus workshop on embryo assessment: proceedings of an expert meeting. Hum Reprod. 2011;26(6):1270-83.

Antczak M, Van Blerkom J. Temporal and spatial aspects of fragmentation in early human embryos: possible effects on developmental competence and association with the differential elimination of regulatory proteins from polarized domains. Hum Reprod. 1999;14(2):429-47.

Barbash-Hazan S, Frumkin T, Malcov M, Yaron Y, Cohen T, Azem F, Amit A, Ben-Yosef D. Preimplantation aneuploidy embryos undergo self-correction in correlation with their developmental potential. Fertil Steril. 2009;92(3):890-6.

Basile N, Vime P, Florensa M, Aparicio Ruiz B, García Velasco JA, Remohí J, Meseguer M. The use of morphokinetics as a predictor of implantation: a multicentric study to define and validate an algorithm for embryo selection. Hum Reprod. 2015;30:276-83.

Bolton H, Graham SJ, Van der Aa N, Kumar P, Theunis K, Fernandez GE, Voet T, Zernicka-Goetz M. Mouse model of chromosome mosaicism reveals lineage-specific depletion of aneuploid cells and normal developmental potential. Nat Commun. 2016;7:11165.

Buehler N, Briton-Jones C, Surrey M, Danzer H, Hill DL. Should morphology assessment include symmetry of blastomeres? The 56th Annual Meeting of the Pacific Coast Reproductive Society, 9-13 April 2008, Rancho Mirage, California, Vol. 89, Issue 4, Supplement, p. S6.

Gardner K, Schoolcraft W. Culture and transfer of human blastocyst. Curr Opin Obstet Gynaecol. 1999;11(3):307-11.

Hardarson T, Hanson C, Sjogren A, Lundin K. Human embryos with unevenly sized blastomeres have lower pregnancy and implantation rates: indications for aneuploidy and multinucleation. Hum Reprod. 2001;16:313-8.

Northrop LE, Treff NR, Levy B, Scott Jr RT. SNP microarray-based 24 chromosome aneuploidy screening demonstrates that cleavagestage FISH poorly predicts aneuploidy in embryos that develop to morphologically normal blastocysts. Mol Hum Reprod. 2010;16(8):590-600.

Prados FJ, Debrock S, Lemmen JG, Agerholm I. The cleavage stage embryo. Hum Reprod. 2012;27(SI):i50-i71.

Puissant F, Van Rysselberge M, Barlow P, Deweze J, Leroy F. Embryo scoring as a prognostic tool in IVF treatment. Hum Reprod. 1987;2:705-8.

Santos MA, Teklenburg G, Macklon NS, Opstal DV, Schuring-Blom GH, Krijtenburg P-J, Vreeden-Elbertse J, Fauser BC, Baart EB. The fate of the mosaic embryo: chromosomal constitution and development of Day 4, 5 and 8 human embryos. Hum Reprod. 2010;25(8):1916-26.

Shenoy CC, Khan Z, Coddington C, Jensen J, Daftary GS, Stewart EA, Morbeck D. Symmetry at the 4-cell stage using time-lapse imaging is correlated with embryo aneuploidy. [Online Poster] 2015.

Sjöblom P, Menezes J, Cummins L, Mathiyalagan B. Prediction of embryo development potential and pregnancy based on early stage morphological characteristics. Fertil Steril. 2006;86(4):848-61.

Soydan E, Esbert M, Ballesteros A, Calderon G. Relationship between day-3 embryo blastomere symmetry and implantation rate. [Online Poster] 2008.

Vergouw CG, Al Nofal M, Kostelijk EH, Rooth H, Hompes PGA, Schats R, Lambalk CB. The association of the blastomere volume index (BVI), the blastomere symmetry index (BSI) and the mean ovality (MO) with ongoing implantation after single embryo transfer. Assist Reprod Technol. 2013;30:587-92.

Wells D, Bermudez MG, Steuerwald N, Malter HE, Thornhill AR, Cohen J. Association of abnormal morphology and altered gene expression in human preimplantation embryos. Fertil Steril. 2005;84(2):343-55.

Ziebe S, Lundin K, Loft A, Bergh C, Nyboe Andersen A, Selleskog U, Nielsen D, GrØndahl C, Kim H, Arce JC, CEMAS II and Study Group. FISH analysis for chromosomes 13, 16, 18, 21, 22, X and $\mathrm{Y}$ in all blastomeres of IVF pre-embryos from 144 randomly selected donated human oocytes and impact on pre-embryo morphology. Hum Reprod. 2003;18(12):2575-81. 